\title{
Analysis of Meiotic Chromosome Behaviour in Diploid Individuals of Chrysanthemum zawadskii and Related Species (Asteraceae): Evidence for Chromosome Rearrangements
}

\author{
Jung Sung Kim ${ }^{1}$, Kazuo Oginuma ${ }^{2}$ and Hiroshi Tobe ${ }^{1, *}$ \\ ${ }^{1}$ School of Life Science and Biotechnology, Division of Life Science, Korea University, \\ Seoul 136-701, Korea. \\ ${ }^{2}$ Department of Environmental Science, Faculty of Human Life and Environmental Science, \\ Kochi Women's University, Kochi 780-8515, Japan. \\ ${ }^{3}$ Department of Botany, Graduate School of Science, Kyoto University, \\ Kyoto 606-8502, Japan.
}

Received November 21, 2008; accepted December 21, 2008

\begin{abstract}
Summary Diploid individuals $(2 n=18)$ of Chrysanthemum zawadskii, C. indicum and C. boreale, two or all three of which often occur together in the wild in East Asia, have diverse metaphase karyotypes. We hypothesised that the diverse karyotypes result from chromosome rearrangements. To test this, we investigated chromosomal behaviour during pollen mother cell (PMC) meiosis and pollen fertility, using 33 individuals collected from seven populations in Korea. All individuals contained one or two multivalents (mostly quadrivalents) at late prophase I and metaphase I in some PMCs (approximately 17-37\%), and at least one or two bivalents composed of homologous chromosomes of different length and centromere position in all PMCs. This meiotic chromosomal behaviour indicates that chromosome rearrangements, i.e., reciprocal translocation and unequal crossing-over, are prevalent in Chrysanthemum species. Nevertheless, because of successful chromosome segregation in anaphase I and II, they produce ample stainable pollen with different chromosome complements. Fertilization by such gametes should result in diverse somatic karyotypes. We also discuss the possibility that older or relatively recent hybridization events have led to a high rate of irregular meiotic chromosome behaviours and pollen sterility, particularly in C. indicum.
\end{abstract}

Key words Chromosome, Chrysanthemum, Hybridization, Karyotype, Meiosis, Rearrangement.

The genus Chrysanthemum (tribe Anthemideae, Asteraceae), which comprises about 37 perennial species that had previously been treated as Dendranthema (Bremer and Humphries 1993, Bremer 1994), is distributed mainly in China, Korea and Japan in East Asia. Some species exhibit notable morphological variation and polyploidy. For instance, C. zawadskii Herbich (=D. zawadskii), which is widely distributed from East Asia through Russia to Europe (Bremer and Humphries 1993), is highly diverse morphologically, and several infraspecies taxa have been proposed (Lee 1996). The species also has a series of polyploids from $2 x$ to $10 x$, with the base number $x=9$ (Kim et al. 2004). In addition, as previously reported (Kim et al. 2003), diploid individuals $(2 n=18)$ of $C$. zawadskii (=Dendranthema zawadskii var. latilobum), C. indicum L. (=D. indicum) and C. boreale Makino $(=D$. boreale) have diverse metaphase karyotypes, not only within species but also within populations. These three species are clearly distinguished by inflorescence and flower characters (Kim et al. 2003). At least two of the three species often occur together in the wild; especially $C$. indicum always grows with $C$. zawadskii and C. boreale and sometimes includes atypical individuals with white ray-flowers ( $c f$. Fig. 2d). Thus, hybridization may have occurred among the species.

\footnotetext{
* Corresponding author, e-mail: tobe@sys.bot.kyoto-u.ac.jp
} 
However, unlike other diploid species $(2 n=18)$ such as Aster iinumae and A. ageratoides which have so distinct karyotypes that can be confirmed in a presumed tetraploid hybrid (Matoba et al. 2007), the three Chrysanthemum species are characterized by the indistinguisbale and diverse karyotypes (Kim et al. 2003).

With regard to karyotype changes, chromosomal rearrangements can alter the size and symmetry of chromosomes, resulting in different karyotypes. These rearrangements include chromosomal fusions and fissions, duplications and deletions of chromosome segments due to unequal crossingover between homologous chromosomes, segment inversions, and segment translocations between non-homologous chromosomes (Stebbins 1971, Raven and Johnson 1999, Rieseberg 2001, Levin 2002). Thus, we hypothesised that the diverse karyotypes in Chrysanthemum species result from chromosome rearrangements. To test this, we investigated chromosome behaviour during PMC meiosis in diploid individuals of $C$. zawadskii, $C$. indicum and $C$. boreale. The diploid species $C$. boreale and C. makinoi Matsum \& Nakai always form nine bivalents at the metaphase I of PMCs (Tanaka 1959a, 1959b, Tanaka and Shimotomai 1961). Any chromosomal rearrangements that did occur should be revealed in unusual chromosome pairings in late prophase I and metaphase I (Stebbins 1971, Levin 2002). For example, chromosomes involved in reciprocal translocation between two non-homologous chromosomes typically form quadrivalents (rings or chains of four chromosomes), while unequal crossing-over should result in bivalents composed of two chromosomes of different length and/or centromere position (Stebbins 1971, Raven and Johnson 1999, Levin 2002). Chromosome rearrangements often generate unbalanced gametes with reduced fertility (Rieseberg 2001, Levin 2002). Thus, we also estimated pollen fertility in these species. Our results will further our understanding of how these species produce fertile gametes to survive and have been sustained in wild populations.

\section{Materials and methods}

\section{Plant materials}

In total, 33 diploid Chrysanthemum individuals $(2 n=18)$ were collected from seven populations (or localities; Fig. 1), including 14 C. zawadskii Herbich, 13 C. indicum K. (including eight atypical individuals with white ray-flowers), and six $C$. boreale Makino (Table 1). These individuals are the same as those we used for karyotype analyses in the previous study (Kim et al. 2003), in which it was shown that almost all of the individuals examined had different karyotypes of somatic chromosomes. As already stated in the introduction, two or all three of the species often occur together as in the Kwangha population (Figs. 2a-f). The plants were planted individually in pots at the Botanical Garden of the Graduate School of Science, Kyoto University (vouchers at KYO).

\section{Meiosis observations}

To obtain PMCs to observe at meiosis, we collected heads with numerous developing flowers from pot-cultivated plants in August-September 2004 and 2005. They were fixed in a ethanol : chloroform : acetic acid $(2: 1: 1)$ solution. PMCs undergoing meiosis were separated with forceps on a glass-slide, stained and macerated in a solution of 10 parts $2 \%$ acetic orcein and one part $1 \mathrm{~N} \mathrm{HCl}$, then smeared and observed with a microscope (Olympus BX51). Chromosome behaviour was analysed at late prophase I, metaphase I and anaphase I, as well as anaphase II and late telophase II.

\section{Pollen fertility}

We estimated pollen fertility by the presence or absence of cytoplasm. Some 1,500 pollen grains from a single head per individual were stained with $1 \%$ aniline blue in lactophenol solution (Shivanna and Rangaswamy 1992). We also measured the sizes (diameter) of stainable and unstainable pollen grains in typical and atypical C. indicum individuals, using 300 grains. 


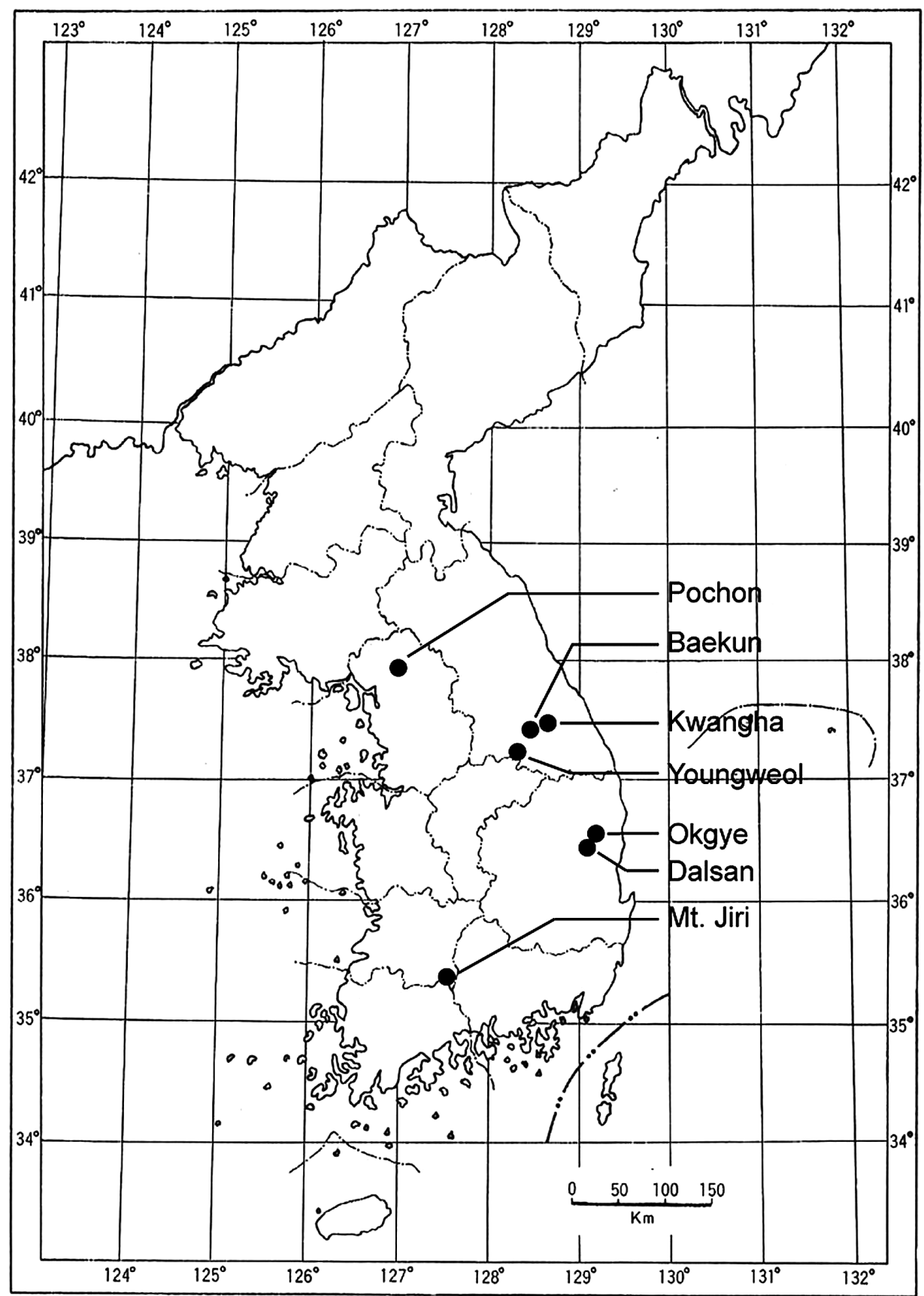

Fig. 1. Map showing the seven sampling sites. Details are: Pochon (Pochon-up, Pochon-kun, Kyunggi-do); Baekun (Baekun-ri, Mitan-myen, Pyungchang-kun, Kangweon-do); Kwangha (Kwangha-ri, Jungsun-up, Jungsun-kun, Kangweon-do); Youngweol (Munkok-myen, Youngweol-kun, Kangweon-do); Okgye (Dalsan-myen, Youngduk-kun, Kyungsangbuk-do); Dalsan (Dalsan-myen, Youngduk-kun, Kyungsangbuk-do); Mt. Jiri (Byuksoryoung, Mt. Jiri-san, Cheollabuk-do). 


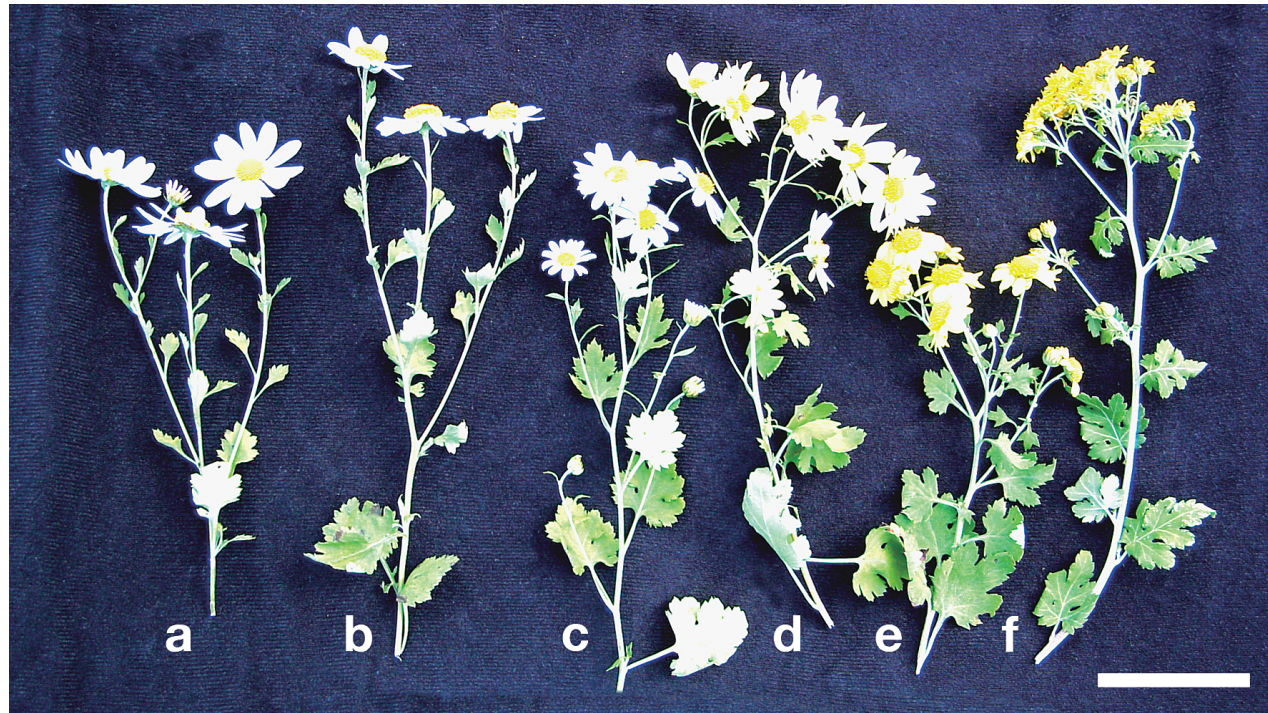

Fig. 2. Diploid Chrysanthemum individuals collected from the Kwangha population. a-c. C. zawadskii. d. Atypical C. indicum, with white ray-flowers. e. Typical C. indicum. f. C. boreale. Scale bar $=5 \mathrm{~cm}$.

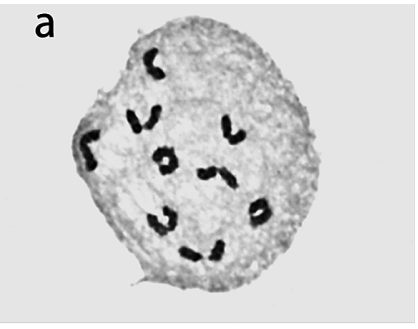

b

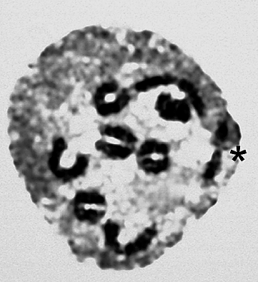

e

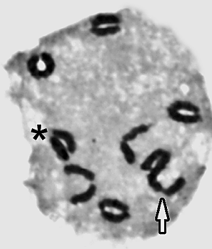

C

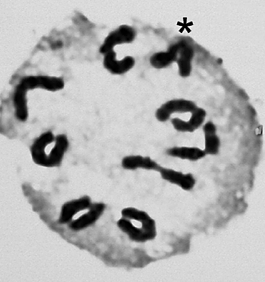

f

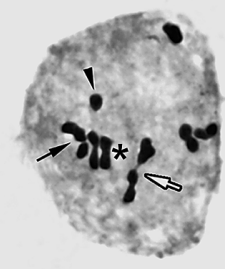

Fig. 3. Micrographs of chromosome configurations at early metaphase I meiotic divisions of pollen mother cells (PMCs) in diploid Chrysanthemum individuals. a-c. PMCs with nine bivalents in C. zawadskii (Kim 312), C. indicum (Kim 318) and C. boreale (Kim 328), respectively. d-f. PMCs containing one or two quadrivalents (white arrows) in C. zawadskii (Kim 307, 313, 311). Note that a bivalent in the rectangle (enlarged at the upper right) is composed of two chromosomes differing in length and centromere position (white arrowheads) in Fig. 3d, and a trivalent (arrow) and univalent (arrowhead) appear in Fig. 3f. Asterisks in Figs. 3b, c, e and $\mathrm{f}$ indicate a pair composed of two chromosomes of different length and centromere position. Scale bar $=10 \mu \mathrm{m}$. 


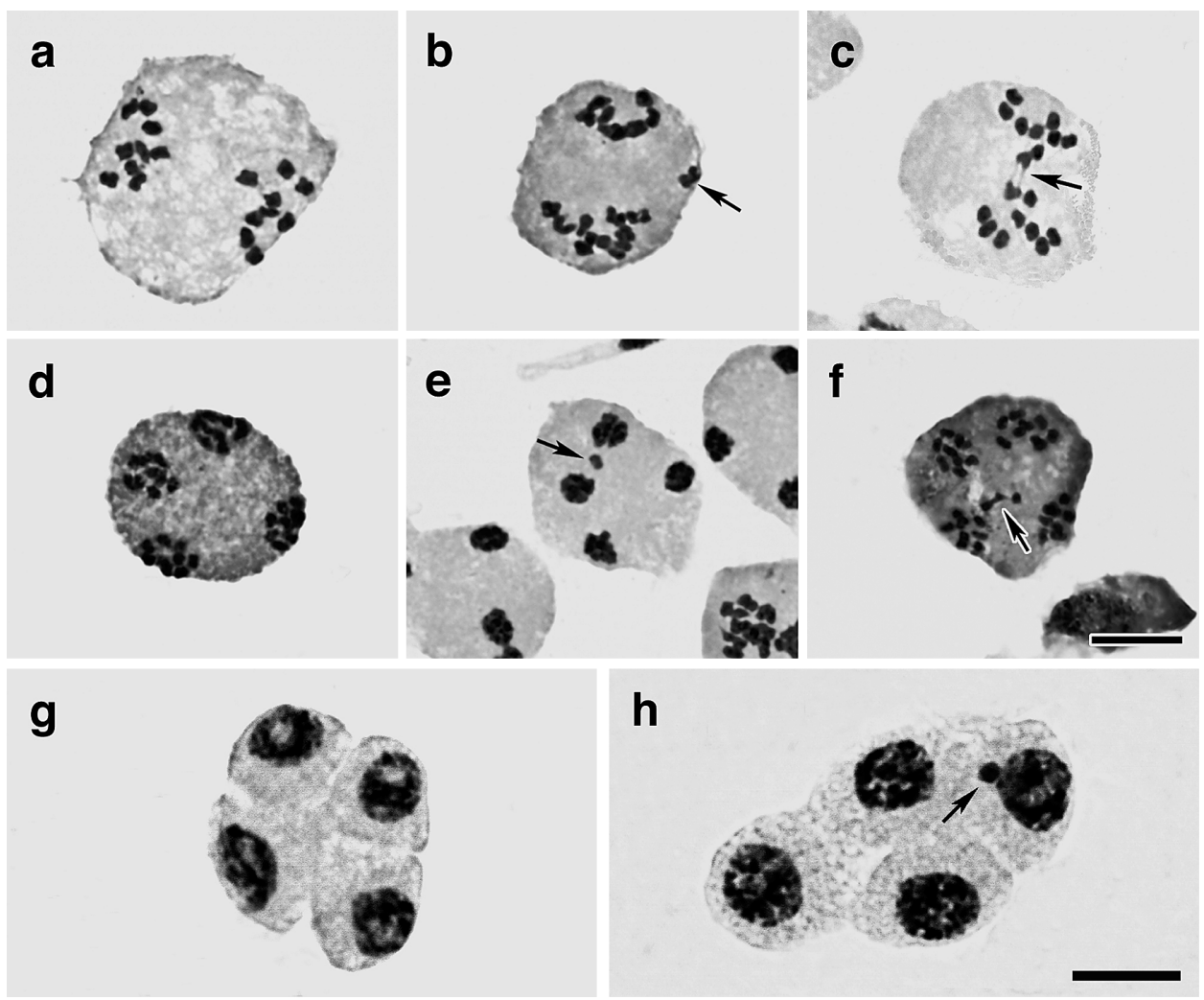

Fig. 4. Micrographs of chromosome segregation in PMCs of Chrysanthemum zawadskii. a-c. At meiotic anaphase I. d-f. At meiotic anaphase II. g and h. Tetrads. Note regular segregation in a, d and g, laggards (arrows) in b and e, chromosome bridges (arrows) in c and $\mathrm{f}$ and a micronucleus (arrow) in $\mathrm{h}$. Scale bars $=10 \mu \mathrm{m}$ (scale bar in $\mathrm{f}$ applies to a-e, and that in $\mathrm{h}$ applies to $\mathrm{g}$ ).

\section{Statistical testing}

We performed analysis of variance (ANOVA) and post hoc multiple comparisons using Scheffe's test $(p<0.05)$ by SPSS 12.0 program to evaluate the significance of differences in the following frequencies among the species: (1) cells with multivalents at late prophase I and metaphase I, (2) cells exhibiting irregular segregation of chromosomes at anaphase I, anaphase II and late telophase II, and (3) stainable pollen grains. The significance of differences in size between stainable and unstainable pollen grains in C. indicum was also evaluated using the $t$-test $(p<0.01)$.

\section{Results}

\section{Chromosome pairing at late prophase I and metaphase I}

In all plants, PMCs with nine bivalents were most often observed at late prophase I and metaphase I (Fig. 3a-c). Every individual had PMCs with creature quadrivalents, appearing as a chain of four chromosomes, in addition to the bivalents. Fig. 3d shows a PMC with two quadrivalents, and Figs. $3 \mathrm{e}$ and $3 \mathrm{f}$ show a PMC with a single quadrivalent. A trivalent was occasionally observed with a univalent (Fig. 3f). In addition, all examined PMCs appeared to contain at least creature bivalents composed of two chromosomes of different lengths and/or centromere position (Fig. 3d, upper right).

The frequency of PMCs with multivalents (quadrivalents and trivalents) varied from 16.90 to 


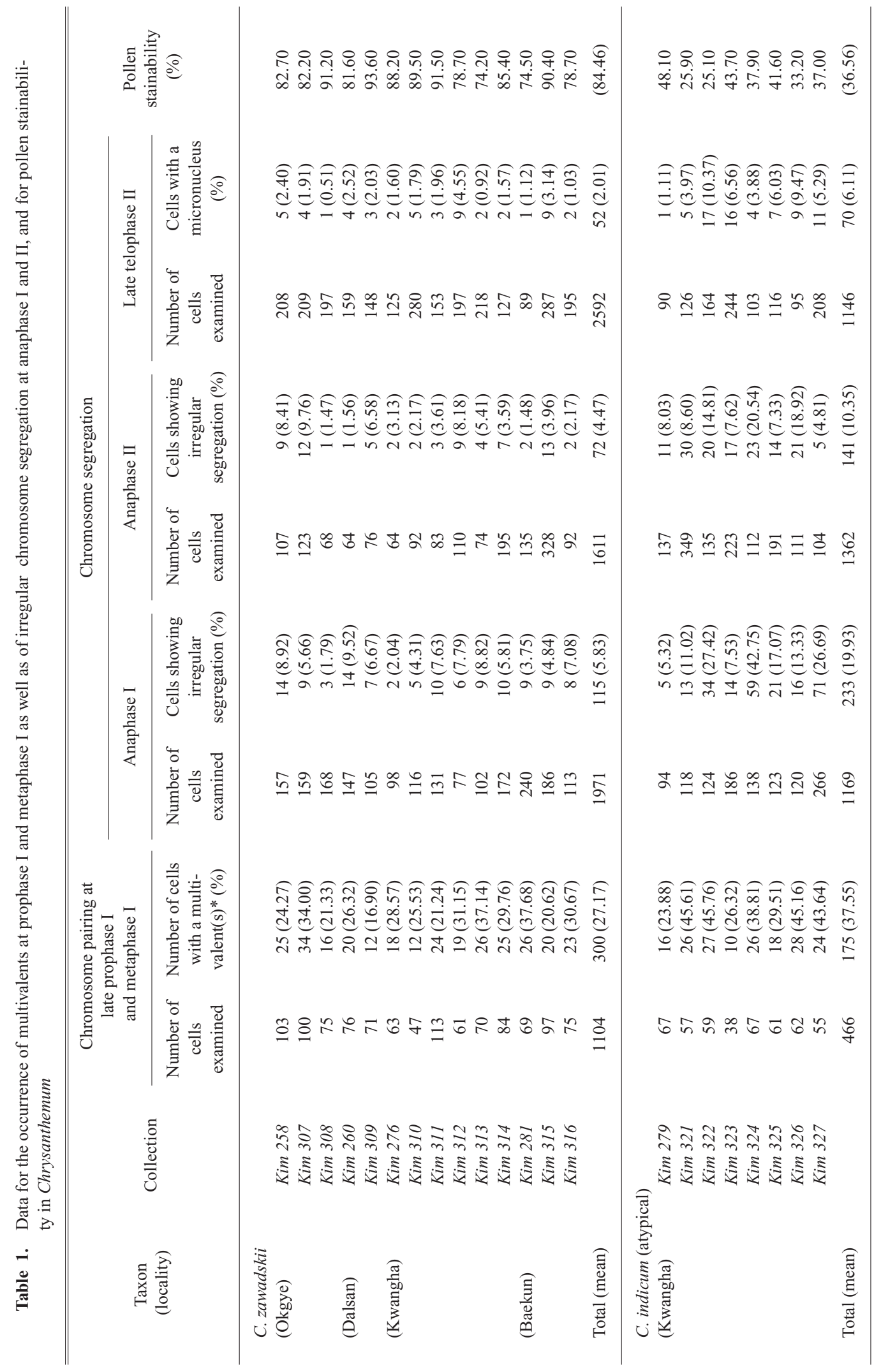




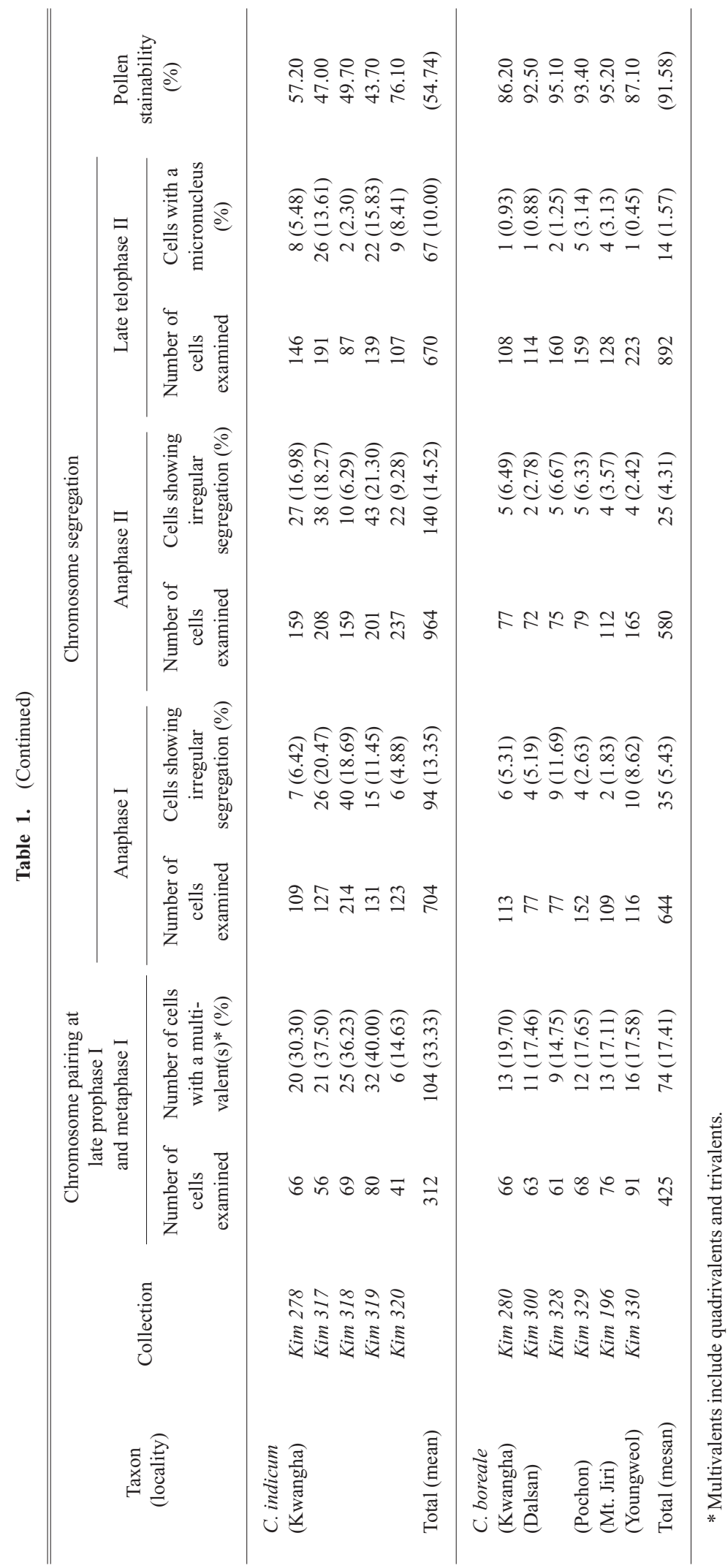


$37.68 \%$ (mean $=27.17 \%)$ in $C$. zawadskii, 23.88 to $45.76 \%$ (mean=37.55\%) in atypical C. indicum, 14.63 to $40.00 \%($ mean $=33.33 \%)$ in typical $C$. indicum, and 14.75 to $19.70 \%$ (mean $=17.41 \%)$ in C. boreale (Table 1). Thus, PMC frequencies with multivalent were highest in atypical C. indicum, lowest in $C$. boreale, and medium in typical $C$. indicum and C. zawadskii. ANOVA showed that a significant difference exists in the mean frequencies of PMCs with multivalents among the species $(p<0.05)$. Scheffe's test further gave a significant difference between $C$. zawadskii and atypical $C$. indicum as well as between $C$. boreale and typical/atypical $C$. indicum .

\section{Chromosome segregation at anaphase I and II and late telophase II}

Chromosomes in most PMCs regularly segregated to opposite poles at anaphase I (Fig. 4a) and anaphase II (Fig. 4d), resulting in four distinct nuclei in late telophase II (Fig. 4g) in all plants. However, parts of chromosomes were often irregularly segregated. As a result, chromosome bridges or laggards were observed at anaphase I (Figs. 4b, c) and/or anaphase II (Figs. 4e, f). One or more micronuclei were also rarely or occasionally observed at late telophase II (Fig. 4h). Frequencies of cells exhibiting such irregular chromosome segregation were relatively low and fairly similar in Chrysanthemum zawadskii and C. boreale. The mean frequencies of cells with irregular chromosome segregation were $5.83 \%$ and $5.43 \%$ at anaphase I, $4.47 \%$ and $4.31 \%$ at anaphase II and $2.01 \%$ and $1.57 \%$ at late telophase II in C. zawadskii and C. boreale, respectively (Table 1). The frequencies were much higher in both typical and atypical $C$. indicum individuals: $13.35 \%$ and $19.93 \%$ at anaphase I, $14.52 \%$ and $10.35 \%$ at anaphase II and $10.00 \%$ and $6.11 \%$ at telophase II in typical and atypical $C$. indicum, respectively (Table 1 ).

Notably, as meiosis proceeded, the frequencies of cells exhibiting irregular chromosome segregation gradually decreased in all species. Nevertheless, at any meiotic stage, the frequency of cells exhibiting irregular chromosome segregation was approximately three to four times higher in both typical and atypical Chrysanthemum indicum than in C. zawadskii and C. boreale. ANOVA showed that the differences in irregular chromosome segregation at any meiotic stage are significant among the species $(p P<0.05)$. Scheffe's test further showed that a significant difference exists at anaphase I between $C$. zawadskii and atypical $C$. indicum as well as between $C$. boreale and atypical $C$. indicum; at anaphase II and late telophase II between C. zawadskii and typical/atypical C. indicum, and C. boreale and typical C. indicum.

\section{Pollen fertility}

Each individual contained both stained and unstained pollen grains at varying frequencies (Figs. 5a-c). Frequencies of stainable pollen grains were the highest (mean $=91.58 \%)$ in C. boreale (Fig. 5c), followed by C. zawadskii (mean $=84.46 \%$, Table 1, Fig. 5a). Stainable pollen grains were less frequent in typical $C$. indicum individuals (mean $=54.74 \%$, Fig. $5 \mathrm{~b}$ ), while atypical $C$. indicum individuals had the lowest frequency of stainable pollen grains (mean $=36.56 \%$, Table 1 ). ANOVA showed that these differences are all significant $(p<0.05)$. Scheffe's test also gave a significant difference between any two of all the species except between $C$. zawadskii and $C$. boreale.

In typical Chrysanthemum indicum, unstainable pollen grains were $17.5-27.5 \mu \mathrm{m}$ (mean \pm standard deviation $=21.79 \pm 1.76$ ) in diameter, and stainable grains were $20.0-32.5 \mu \mathrm{m}$ (24.18 \pm 1.90$)$. Likewise, in atypical $C$. indicum, unstainable pollen grains were $8.75-30.0 \mu \mathrm{m}$ $(22.63 \pm 2.17)$ and stainable grains were $22.5-37.5 \mu \mathrm{m}(26.32 \pm 1.97)$. The smaller size of unstainable pollen grains was significant $(t$-test: $p<0.01)$.

\section{Discussion}

\section{Chromosome rearrangement and diverse karyotypes}

Some PMCs (approximately 17-37\%, see Table 1) in all 33 plants of C. zawadskii, C. indicum 
(including atypical individuals with white ray-flowers) and $C$. boreale contained bivalents and multivalents (mostly quadrivalents and occasionally trivalents) at late prophase I and metaphase I, indicating that reciprocal translocation between two non-homologous chromosomes had occurred. Also, the bivalents included at least one or two distinct bivalents, with the two chromosomes differing in length and/or centromere position. As the presence of such bivalents does not hinder chromosome segregation in later meiotic stages, they were likely formed by an unequal crossing-over between homologous chromosomes, as reported in other angiosperm taxa, including Viscum (Soman and Bhavanandan 1993). Thus, chromosome rearrangements, such as reciprocal translocations and unequal crossing-over, appear to be prevalent in the three Chrysanthemum species, resulting in gametes with different chromosome complements.

Chromosomal rearrangements are likely to reduce pollen fertility (Levin 2002), because of the failure of regular chromosome segregation in later meiotic stages. However, in the three Chrysanthemum species, the frequencies of cells exhibiting irregular chromosome segregation gradually decreased as the meiotic stages proceeded (Table 1). Particularly in C. zawadskii and C. boreale, the frequencies of cells exhibiting irregular chromosome segregation were far less than those of cells with multivalents (quadrivalents and trivalents; Table 1), indicating that even cells with multivalents generally undergo regular chromosome segregation at anaphase I and II to produce stainable pollen. Among other angiosperm species, Convallaria keiskei (Ruscaceae) exhibits 52.2\% pollen fertility, despite the high frequency of cells with quadrivalents (>94\%; Noda and Zhu 1996). In C. keiskei, almost half of the cells with quadrivalents undergo normal chromosome disjunction to produce fertile pollen. Thus, with respect to chromosome segregation, Chrysanthemum species appear to go through meiotic division similarly to $C$. keiskei, producing fertile pollen with high genetic diversity in wild populations. Fertilization by these gametes should result in diverse somatic karyotypes, as found in our previous study (Kim et al. 2003).

\section{Hybridization events in Chrysanthemum}

Among the three species examined, the frequency of laggard chromosomes, chromosome bridges, and/or micronuclei was highest in C. indicum, which resulted in irregular chromosome segregation at much higher frequencies at anaphase I and II and late telophase II than in the other species (Table 1). C. indicum also showed a much higher rate of unstainable pollen than did C. $z a-$ wadskii or C. boreale (Table 1), and the unstainable pollen grains were smaller than stainable pollen grains. Although we did not pursue the fate of laggard chromosomes in these plants, in other angiosperm species, including Tribulus spp. (Husain 1986) and Avena sativa L. (Baptista-Giacomelli et al. 2000), laggard chromosomes form micronuclei, which develop into nonfunctional dwarf pollen. This may be the case in $C$. indicum. Laggards and micronuclei, which were found at the highest frequencies during meiosis, appeared to develop into small-sized unstainable pollen grains, resulting in a high rate of unstainable pollen in $C$. indicum.

Why do both typical and atypical C. indicum have such high rates of unstainable pollen? C. indicum always occurs with $C$. zawadskii and $C$. boreale in populations. In angiosperms interspecific hybridization causes irregular meiosis and/or a high rate of pollen sterility (Saitwal et al. 2003). C. indicum has been regarded as a distinct taxon (Lee 1996), but it may have originated as a hybrid between $C$. zawadskii and C. boreale. However, diploid C. indicum individuals may be old and long established. They appear to set some seeds, although we have not confirmed their viability. Atypical C. indicum individuals have white ray-flowers, as does C. zawadskii, and may represent a recent cross with C. zawadskii. They are morphologically intermediate between C. zawadskii and C. indicum.

Some wild populations are composed only of diploid C. zawadskii and C. boreale individuals. These species also exhibit lower but considerably high rates of PMCs with multivalents and irregular chromosome segregation. There is the possibility that older hybridization events have led to such 

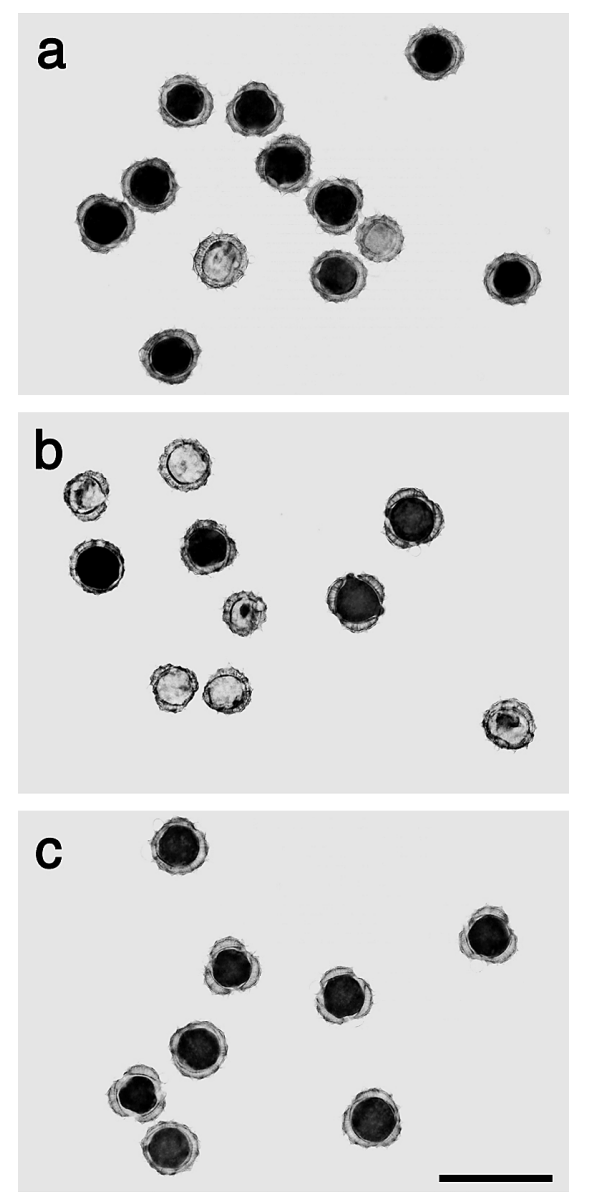

Fig. 5. Micrographs of pollen grains from diploid Chrysanthemum individuals. Stained grains by aniline blue are estimated to be fertile. a. C. $z a$ wadskii (Kim 309). b. C. indicum (Kim 319). c. C. boreale (Kim 280). Scale bar $=50 \mu \mathrm{m}$. chromosome behaviours in C. zawadskii and $C$. boreale. We suspect that in those populations, $C$. indicum may be generated in the future by hybridization between $C$. zawadskii and $C$. boreale. On the other hand, it may already have been extirpated, owing to high pollen sterility. Fertility in hybrids is restored by polyploidization (e.g., Isshiki and Taura 2003, Pushpam and Raveendran 2006). In other East Asian areas, C. indicum now grows as a tetraploid or hexaploid (Taniguchi 1987). Recently, recurrent origins of tetraploid C. indicum in China were suggested by molecular evidence (Yang et al. 2006). The aforementioned homoploid hybridization events, which were suspected to have occurred in diploid Chrysanthemum species, may also be confirmed by a molecular study using DNA sequences as recently attempted by Kim et al. (2008) in Sonchus species (Asteraceae) or species-specific markers (for review of current researches see Hegarty and Hiscock 2005).

\section{Acknowledgements}

We are grateful to Jae-Hong Pak and his students for their assistance in collecting materials used for this study. The study was supported in part by a Grant-in-Aid for Scientific Research from the Japan Society for the Promotion of Science (No. 18370036).

\section{References}

Baptista-Giacomelli, F. R., Pagliarini, M. S. and Almeida, J. L. 2000. Elimination of micronuclei from microspores in a Brazilian oat (Avena sativa L.) variety. Gen. Mol. Biol. 23: 681-684.

Bremer, K. 1994. Asteraceae: Cladistics and Classification. Timber Press, Portland.

— and Humphries, C. 1993. Generic monograph of the Asteraceae-Anthemideae. Bull. Brit. Mus. (Nat. Hist.), Bot. 23: 7 3-177.

Hegarty, M. J. and Hiscock, S. J. 2005. Hybrid speciation in plants: new insights from molecular studies. New Phytol. 165: 411-423.

Husain, S. A. 1986. Cytotaxonomic studies in Tribulus from Pakistan. La Kromosomo II 42: 1316-1329.

Isshiki, S. and Taura, T. 2003. Fertility restoration of hybrids between Solanum melongena L. and S. aethiopicum L. Gilo group by chromosome doubling and cytoplasmic effect on pollen fertility. Euphytica 134: 195-201.

Kim, J.-S., Pak, J.-H., Seo, B.-B. and Tobe, H. 2003. Karyotypes of metaphase chromosomes in diploid populations of Dendranthema zawadskii and related species (Asteraceae) from Korea: diversity and evolutionary implications. J. Plant Res. 116: 47-55.

- - - and Tobe, H. 2004. Chromosome number of Dendranthema coreana (Asteraceae). Acta Phytotax. Geobot. 55: 63-64. 
Kim, S.-C., Mejías, J. A. and Lubinsky, P. 2008. Molecular confirmation of the hybrid origin of the critically endangered western Mediterranean endemic Sonchus pustulatus (Asteraceae: Sonchinae). J. Plant Res. 121: 357-364.

Lee, Y. N. 1996. Flora of Korea. Kyohaksa, Seoul.

Levin, D. A. 2002. The Role of Chromosomal Change in Plant Evolution. Oxford Univ. Press, Oxford.

Matoba, H., Soejima, A. and Hoshi, Y. 2007. Identification of parental genomes and genomic organization in Aster microcephalus var. ovatus. J. Plant Res. 120: 585-593.

Noda, S. and Zhu, U.-C. 1996. Cytogenetics of Liliaceae in East Asia. II. External morphology, karyotype, and reciprocal translocation of Convallaria keiskei in North-East district of China. La Kromosomo II 83-84: 2909-2919 (in Japanese with English summary).

Pushpam, R. and Raveendran, T. S. 2006. Production of interspecific hybrids between Gossypium hirsutum and Jassid resistant wild species G. raimondii and G. armourianum. Cytologia 71: 407-418.

Raven, P. H. and Johnson, G. B. 1999. Biology. 5th ed. McGraw-Hill, Boston.

Rieseberg, L. H. 2001. Chromosome rearrangements and speciation. Trends Ecol. Evol. 16: 351-358.

Saitwal, V. M., Mehetre, S. S., Sonone, A. H. and Gawande, V. L. 2003. Cytomorphology of F1, F2, backcross generations of interspecific cross between Gossypium arboretum $\times$ Gossypium anomalum. Cytologia 68: 317-327.

Shivanna, K. R. and Rangaswamy, N. S. 1992. Pollen Biology—a Laboratory Manual. Springer, Berlin.

Soman, T. A. and Bhavanandan, K. V. 1993. Cytological studies in Indian Viscaceae: I. Chromosome study of karyotype analysis in Viscum capitellatum Sm. Caryologia 46: 227-231.

Stebbins, G. L. 1971. Chromosomal Evolution in Higher Plants. Edward Arnold Ltd., London.

Tanaka, R. 1959a. On the speciation and karyotypes in diploid and tetraploid species of Chrysanthemum I. Karyotypes in Chrysanthemum boreale $(2 n=18)$. J. Sci. Hiroshima Univ., Ser B., Div. 29: 1-15.

- 1959b. On the speciation and karyotypes in diploid and tetraploid species of Chrysanthemum II. Karyotypes in Chrysanthemum makinoi $(2 n=18)$. J. Sci. Hiroshima Univ., Ser B., Div. 29: 17-30.

— and Shimotomai, N. 1961. Karyotypes in four diploid species of Chrysanthemum. Cytologia 26: 309-319.

Taniguchi, K. 1987. Cytogenetical studies on the speciation of tetraploid Chrysanthemum indicum L. with special reference to C-bands. J. Sci. Hiroshima Univ., Ser B., Div. 221: 105-157.

Yang, W., Glover, B. J., Rao, G.-Y. and Yang, J. 2006. Molecular evidence for multiple polyploidization and lineage recombination in the Chrysanthemum indicum polyploid complex (Asteraceae). New Phytol. 171: 875-886. 